\title{
Dr. Roberto Mioso (1965-2018): uma vida, um legado
}

\author{
Silvio Felipe Barbosa Lima ${ }^{1,2}$ (I)
}

(1) Universidade Federal de Campina Grande, Centro de Formação de Professores, Unidade Acadêmica de Ciências Exatas e da Natureza, Rua Sérgio Moreira de Figueiredo, Casas Populares, Cajazeiras 58900000, Paraíba, Brasil. E-mail: silvio.lima@ufcg.edu.br

(2) Universidade Federal da Paraíba - Campus I, Centro de Ciências Exatas e da Natureza, Departamento de Sistemática e Ecologia, Programa de Pós-Graduação em Ciências Biológicas (Zoologia), Cidade Universitária 58051-900, João Pessoal, Paraíba, Brasil. E-mail: sfblima@gmail.com

Lima S.F.B. (2020) Dr. Roberto Mioso (1965-2018): uma vida, um legado. Pesquisa e Ensino em Ciências Exatas e da Natureza, 4: e1297. http://dx.doi.org/10.29215/pecen.v4i0.1297

Dr. Roberto Mioso nasceu em 05 de março de 1965, em São Luis Gonzaga, município da região das Missões, noroeste do estado do Rio Grande do Sul, Brasil (Figura 1). Ele obteve o grau acadêmico de bacharel em Oceanologia (Oceanografia Biológica) pela Universidade Federal do Rio Grande (FURG: 1983-1988), Brasil. Entre 1990 e 1991, Roberto realizou curso técnico no Politecnico Internazionale per lo Sviluppo Industriale ed Economico (PISIE), Itália, financiado com bolsa do Ministério das Relações Exteriores da Itália. Ele obteve o grau acadêmico de mestre em Aquicultura pela Universidade Federal de Santa Catarina (UFSC: 1990-1995), Brasil, defendendo a dissertação intitulada "Indução à reprodução e incubação de ovos do robalo Centropomus parallelus poey, 1860 (Pisces, Centropomidae)”, sob orientação do Dr. Vinicius Ronzani Cerqueira, e entre 1994 e 1995, concomitantemente, foi assessor do Programa de Piscicultura da Fundação Municipal de Desenvolvimento Rural (FMDR), Brasil, implantando novas técnicas e sistemas de reprodução de peixes de água-doce, assessorando a formação, manutenção e seleção de reprodutores, bem como a produção de larvas no laboratório e alevinos nos viveiros. Além disso, ele implantou novas técnicas de arraçoamento e manejo alimentar no sistema produtivo, prestando assistência técnica aos produtores e transferindo tecnologia aos funcionários. Roberto também atuou como Pesquisador Visitante da University of Aberdeen (ABDN: 2001-2002), Escócia, sob a supervisão do Dr. Marcel Jaspars. Obteve o grau acadêmico de doutor em Recursos Pesqueiros e Aquicultura pela Universidad de Las Palmas de Gran Canaria (ULPGG: 2007-2010), Espanha, defendendo a tese intitulada "Productos Naturales de Schizochytrium aggregatum, Paecilomyces variotii y Penicillium roqueforti, hongos de interés acuícola”, sob a orientação do Dr. Francisco Javier Toledo Marante. Foi bolsista da Coordenação de Aperfeiçoamento de Pessoal de Nível Superior (CAPES) durante o Mestrado e o Doutorado. Em 2011, Roberto passou a morar no Nordeste do Brasil para a realização de Pós-doutorado pela Universidade Federal da Paraíba (UFPB: 2011-2013), Brasil. Posteriormente, ele ingressou em outros dois pós-doutorados no Nordeste do país, um pela Universidade Federal de Sergipe (UFS: 2013-2014) e outro pela Universidade Federal de Pernambuco (UFPE: 2015-2017). Em 2017, Roberto retornou à ULPGG para atuar como Pesquisador Associado Externo em seu quarto e último pós-doutoramento.

Durante sua atuação como Pesquisador Associado Externo da ULPGC, em junho de 2018, em Las Palmas de Gran Canaria, capital da ilha de Gran Canaria, Espanha, conforme informação da sua ex-esposa, Roberto Mioso faleceu, vítima de um câncer, deixando uma considerável produção intelectual. Ele foi bolsista do Conselho Nacional de Desenvolvimento Científico e Tecnológico (CNPq) durante a realização dos pós-doutorados no Nordeste do Brasil e no exterior. 


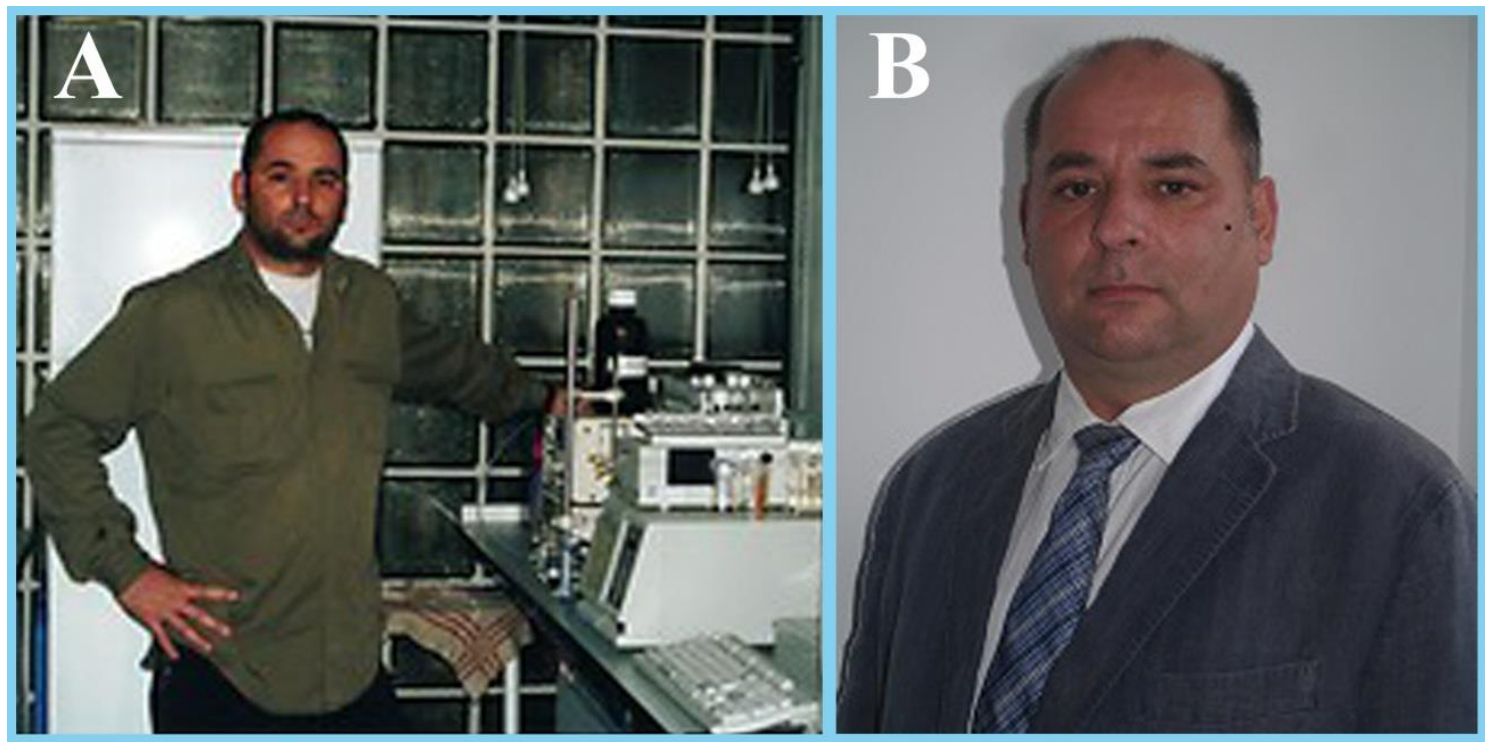

Figura 1. Fotografias do Dr. Roberto Mioso (1965-2018) em épocas e localidades desconhecidas. Fonte: A. Erasmusu.com (2020); B. IUNAT (2020).

Roberto atuou nas seguintes áreas do conhecimento: (1) Ciências Agrárias: Recursos Pesqueiros e Engenharia de Pesca: Aquicultura, Piscicultura, Maricultura; (2) Ciências Exatas e da Terra: Química: Química Orgânica: Química dos Produtos Naturais; e (3) Ciências Biológicas: Zoologia; Microbiologia: Microbiologia Aplicada: Microbiologia Industrial e de Fermentação.

Roberto atuou como revisor de 18 periódicos internacionais (African Journal of Biotechnology, African Journal of Microbiology Research, BMC Microbiology, Chemical Science International Journal, Current Bioactive Compounds, Current Traditional Medicine, Dynamic Journal of Pure and Applied Microbiology, Issues in Biological Sciences and Pharmaceutical Research, International Journal of Agricultural Policy and Research, Journal of Chemical Technology and Biotechnology, Journal of Ecology and The Natural Environment, Letters in Applied Microbiology, Letters in Drug Design \& Discovery, Marine Drugs, Nutrients, Patent eBook Series e Plant Disease and Preparative Biochemistry and Biotechnology) e um nacional (Química Nova) nas áreas de Agronomia, Biotecnologia, Bioquímica, Ecologia, Farmacologia, Medicina, Microbiologia e Química.

Em 1993, Roberto publicou o seu primeiro artigo sobre o crescimento de alevinos de tainha, Mugil platanus, da Ilha de Santa Catarina (Cerqueira et al. 1993). Posteriormente, outros dois artigos foram publicados na mesma linha de pesquisa, só que envolvendo o peixe linguado (Paralichthys orbignyanus) e robalo-peva (Centropomus parallelus), respectivamente (Cerqueira et al. 1995, 2005). Depois disso, publicou quase que exclusivamente sobre compostos bioativos de fungos, microalgas e esponjas, temáticas que prevaleceram até a sua morte em 2018. Em 2012, Roberto publicou o seu primeiro artigo envolvendo fungos, intitulado "Caracterização estrutural e perfil metabólico do fungo marinho Paecilomyces variotii (Toledo Marante et al. 2012). Os anos de 2014 e 2015 foram os mais produtivos na carreira do Oceanólogo com a publicação de seis artigos científicos, em cada ano, envolvendo a identificação de compostos orgânicos bioativos dos fungos Penicillium roqueforti (Mioso et al. 2014a, 2015a,b; Toledo Marante et al. 2014) e Paecilomyces variotii (Bravo de Laguna et al. 2015a; Mioso et al. 2015c) e das microalgas Schizochytrium sp. (Mioso et al. 2014b) e Spirulina (Bravo de Laguna et al. 2015b), empregando técnica de cromatografia (Mioso et al. 2014c; Bravo de Laguna et al. 2015b). Roberto também publicou um artigo sobre a associação entre o caranguejo Dissodactylus crinitichelis (Decapoda, Pinnotheridae) e a bolacha-da-praia Mellita quinquiesperforata com base em indivíduos coletados na costa da Paraíba (Nordeste do Brasil) (Lima et al. 2014) e elaborou dois artigos de revisão da literatura, um sobre a química de produtos naturais aplicada à aquicultura (Mioso et al. 2014d) e outro sobre as características estruturais da proteína "lectina" derivada de esponjas 
marinhas (Gomes Filho et al. 2015). Em 2016, Roberto publicou sobre a atividade antileishmaniose do composto dehidrodieugenol contra Leishmania amazonenses (Rodrigues et al. 2016); e o metabólito bioativo "Forscolina" proveniente da raiz da planta Coleus forskohlii, que foi extraído por meio de um procedimento simples e eficaz usando cromatografia e seus componentes caracterizados por modernas técnicas espectroscópicas (Mioso et al. 2016). Em 2017, ele publicou sobre a "Formação de benzofurano bioativo via acoplamento oxidativo, utilizando água de coco (Cocos nucifera L.) como biocatalisador" (Rodrigues et al. 2017) e “Compostos citotóxicos derivados de esponjas marinhas" (Mioso et al. 2017). Em 2018, ele também voltou a publicar sobre compostos extraídos a partir de esponjas marinhas (Ferriol et al. 2018) e sobre a "Síntese de ésteres fenilpropanóides naturais através de reações químicas convencionais" (Borges et al. 2018).

Roberto Mioso participou de 22 eventos científicos nacionais e internacionais, atuou em 6 projetos de pesquisa e como revisor de projeto de fomento para a FACEPE e Fundação de Apoio à Pesquisa e à Inovação Tecnológica do Estado de Sergipe (FAPITEC), além de ter registrado 8 patentes e coorientado uma dissertação de Mestrado em Produtos Naturais e Sintéticos Bioativos pela UFPB entre 2012 e 2014.

Em 2012, eu conheci Roberto Mioso durante a realização do meu doutorado no Departamento de Sistemática e Ecologia da UFPB. Naquele ano, ele estava realizando o seu pósdoutorado também pela UFPB. Desde então, nos tornamos grande amigos e realizamos algumas coletas no litoral da Paraíba. Roberto era um cientista criativo, inteligente, repleto de ideias e curiosidades, sempre disposto a enfrentar novos desafios científicos. Roberto era pra mim, acima de tudo, um amigo. Infelizmente, ele faleceu aos 53 anos, mas sempre permanecerá vivo na memória de seus amigos e colegas.

Um grande abraço, meu irmão.

\section{Agradecimentos}

A Diretoria Científica da Fundação de Amparo à Ciência e Tecnologia do Estado de Pernambuco (FACEPE) por repassar informações sobre Roberto Mioso. A Prof ${ }^{a}$ Dr $^{a}$ Maria Nazareth de Lima Arrais (Universidade Federal de Campina Grande, Centro de Formação de Professores, Unidade Acadêmica de Letras) pela revisão gramatical cuidadosa e sugestões ao texto. Todas as informações aqui apresentadas sobre a trajetória de Roberto Mioso foram retiradas do seu Curriculo Lattes (http://lattes.cnpq.br/3122504986109532).

\section{Referências}

Borges F.V.P., Mioso R., Silva L.A.A., Barbosa Filho J.M., Duarte G.D. \& Rodrigues L.C. (2018) Synthesis of natural phenylpropanoid esters via conventional chemical reactions. Organic Communications, 11(1): 53-61. https://doi.org/10.25135/acg.oc.39.18.02.065

Bravo de Laguna I.H, Toledo Marante F.J. \& Mioso R. (2015a) Enzymes and bioproducts produced by the ascomycete fungus Paecilomyces variotii. Journal of Applied Microbiology, 119(6): 1455-1466. https://doi.org/10.1111/jam.12934

Bravo de Laguna I.H, Toledo Marante F.J., Luna-Freire K.R. \& Mioso R. (2015b) Extraction of nutraceuticals from Spirulina (blue-green alga): A bioorganic chemistry practice using thinlayer chromatography. Biochemistry and Molecular Biology Education, 43(5): 366-369. https://doi.org/10.1002/bmb.20882

Cerqueira V.R., Mioso R., Macchiavello J.A.G. \& Brugger A.M. (1993) Observações sobre o crescimento de alevinos de tainha, Mugil platanus, da Ilha de Santa Catarina, com uma dieta rica em proteína. Revista Acovez, 7: 14-17.

Cerqueira V.R., Mioso R., Macchiavello J.A.G. \& Brugger A.M. (1997) Ensaios de indução de desova do linguado (Paralichthys orbignyanus Valenciennes, 1839). Boletim do Instituto de Pesca, 24: 247-254. 
Cerqueira V.R., Mioso R. \& Canarin M. (2005) Indução de desova com fertilização natural e artificial e incubação de ovos do robalo-peva (Centropomus parallelus). Atlântica, 27(1): 3138.

Erasmusu.com (2020) Roberto Mioso. Erasmusu.com. Disponível em: https://erasmusu.com/pt/ro berto-mioso-682925 (Acessado em: 05/01/2020).

Ferriol P., Toledo Marante F.J., Martin I.B., Rodríguez J.J.S., Alonso R.G., Benkovics A.G. \& Mioso R. (2018) Identification and Quantification, by NMR and LC-MS, of Sterols Isolated from the Marine Sponge Aplysina aerophoba. Records of Natural Products, 12(5): 470-479. http://doi.org/10.25135/rnp.52.17.11.558.204

Gomes Filho S.M., Cardoso J.D., Lopes K.A.J., Nascimento E.S., Lacerda J.T.J.G., Mioso R., Gadelha T.S. \& Gadelha C.A.A. (2015) Marine Sponge Lectins: Actual Status on Properties and Biological Activities. Molecules, 20(1): 348-357. http://doi.org/10.3390/molecules20010348

IUNAT (2020) Roberto Mioso: Associate Researcher. Instituto de Estudios Ambientales y Recursos Naturales (IUNAT). Universidad de Las Palmas de Gran Canaria (ULPGC). Disponível em: http://www.iunat.ulpgc.es/iunat-members-lectures (Acessado em: 05/01/2020).

Lima S.F.B., Queiroz V., Bravo de Laguna I.H. \& Mioso R. (2014) New host for Dissodactylus crinitichelis (Decapoda: Pinnotheridae): First record of occurrence on Mellita quinquiesperforata (Echinodermata: Echinoidea). Spixiana, 37: 61-68.

Mioso R., Toledo Marante F.J., Bravo de Laguna I.H., Gonzalez J.E.G. \& Rodriguez J.J.S. (2014a) Biomolecules produced in liquid-state fermentation by a marine-derived fungus. Química Nova, 37(2): 260-267. http://dx.doi.org/10.5935/0100-4042.20140045

Mioso R., Toledo Marante F.J., Gonzalez J.E.G., Rodriguez J.J.S. \& Bravo de Laguna I.H. (2014b) Metabolite profiling of Schizochytrium sp by GC-MS, an oleaginous microbial source of biodiesel. Brazilian Journal of Microbiology, 45(2): 403-409. http://dx.doi.org/10.1590/S151783822014000200006

Mioso R., Bravo de Laguna I.H., Toledo Marante F.J. \& Barbosa Filho J.M. (2014c) Recycled X-ray films as an alternative support for thin-layer chromatography plates. JPC. Journal of Planar Chromatography, Modern TLC, 27(4): 315-317. http://dx.doi.org/10.1556/JPC.27.2014.4.12

Mioso R., Toledo Marante F.J., Bravo de Laguna I.H. \& Bessonart M. (2014d) Natural products chemistry applied to aquaculture: an interdisciplinary review. Química Nova, 37(3): 513-520. http://dx.doi.org/10.5935/0100-4042.20140084

Mioso R., Toledo Marante F.J. \& Bravo de Laguna I.H. (2015a) Chemical constituents of the fermentation broth of the marine-derived fungus Penicillium roqueforti. Revista Iberoamericana de Micología, 32(3): 147-152. http://dx.doi.org/10.1016/j.riam.2014.01.004

Mioso R., Toledo Marante F.J. \& Bravo de Laguna I.H. (2015b) Penicillium roqueforti: A multifunctional cell factory of high value-added molecules. Journal of Applied Microbiology, 118(4): 781-791. http://dx.doi.org/10.1111/jam.12706

Mioso R., Toledo Marante F.J. \& Bravo de Laguna I.H (2015c) The Chemical Diversity of the Ascomycete Fungus Paecilomyces variotii. Biotechnology and Applied Biochemistry, 177(4): 781-791. http://dx.doi.org/0.1007/s12010-015-1783-z

Mioso R., Toledo Marante F.J. \& Bravo de Laguna I.H. (2016) Simple screening method for the detection and isolation of forskolin and related terpenes from the crude plant extract of Coleus forskohlii using chromatography. Revista de la Academia Canaria de Ciencias, 28: 8998.

Mioso R., Toledo Marante F.J., Bezerra R.S., Borges F.V.P., Santos B.V.O. \& Bravo de Laguna I.H. (2017) Cytotoxic Compounds Derived from Marine Sponges. A Review (2010-2012). Molecules, 22(2): 208-242. http://dx.doi.org/10.3390/molecules22020208

Rodrigues L.C., Barbosa Filho J.M., Oliveira M.R., Neris P.L.N., Borges F.V.P. \& Mioso R. (2016) Synthesis and Antileishmanial Activity of Natural Dehydrodieugenol and Its Mono and Dimethyl Ethers. Chemistry \& Biodiversity, 13(7): 870-874. http://dx.doi.org/10.1002/cbdv.201500280

Rodrigues L.C., Barbosa-Filho J.M., Marques S.D.G., Borges F.V.P., Silva L.A.A., Bravo de Laguna I.H. \& Mioso R. (2017) Formation of bioactive benzofuran via oxidative coupling, using 
coconut water (Cocos nucifera L.) as biocatalyst. Organic Communications, 10(2): 72-78. http://doi.org/10.25135/acg.oc.10.16.11.449

Toledo Marante F.J., Bravo de Laguna I.H., Torres N.V. \& Mioso R. (2014) Evidence of a New Intermediate Compound of the Chitin Biogenesis Found in a Marine-Derived Fungus. Applied Biochemistry and Biotechnology, 174: 2426-2434. http://doi.org/10.1007/s12010-0141180-z

Toledo Marante F.J., Mioso R., Barrera J.B., González J.E.G., Rodríguez J.J.S. \& Bravo de Laguna I.H. (2012) Structural characterization and metabolite profiling of the facultative marine fungus Paecilomyces variotii. Annals of Microbiology, 62: 1601-1607.

http://doi.org/10.1007/s13213-011-0416-1 